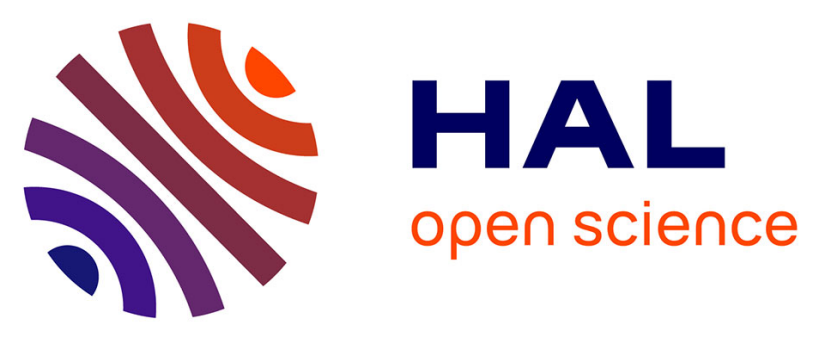

\title{
Simplification in the Acquisition and Analysis of Fluorescence Decays Acquired with Polarized Emission for Time-Resolved Fluorescence Anisotropy Measurements
}

Hunter Little, Jingqi Wang, Jean Duhamel, Xuesong Li, Nagula Markandeya, Victor Maurizot, Ivan Huc

\section{To cite this version:}

Hunter Little, Jingqi Wang, Jean Duhamel, Xuesong Li, Nagula Markandeya, et al.. Simplification in the Acquisition and Analysis of Fluorescence Decays Acquired with Polarized Emission for TimeResolved Fluorescence Anisotropy Measurements. Analytical Chemistry, 2019, 92 (1), pp.668-673. 10.1021/acs.analchem.9b05021 . hal-03043098

\section{HAL Id: hal-03043098 https://hal.science/hal-03043098}

Submitted on 7 Dec 2020

HAL is a multi-disciplinary open access archive for the deposit and dissemination of scientific research documents, whether they are published or not. The documents may come from teaching and research institutions in France or abroad, or from public or private research centers.
L'archive ouverte pluridisciplinaire HAL, est destinée au dépôt et à la diffusion de documents scientifiques de niveau recherche, publiés ou non, émanant des établissements d'enseignement et de recherche français ou étrangers, des laboratoires publics ou privés. 
Simplification in the Acquisition and Analysis of Fluorescence Decays Acquired with Polarized Emission for Time-Resolved Fluorescence Anisotropy Measurements

\author{
Hunter Little, ${ }^{1}$ Jingqi Wang, ${ }^{1}$ Jean Duhamel, ${ }^{1 *}$ \\ Xuesong Li, ${ }^{2}$ Nagula Markandeya, ${ }^{2}$ Victor Maurizot, ${ }^{2}$ Ivan Huc ${ }^{2,3}$ \\ ${ }^{1}$ Institute for Polymer Research, Waterloo Institute for Nanotechnology, Department of \\ Chemistry, University of Waterloo, 200 University Avenue West, \\ Waterloo, ON N2L 3G1, Canada \\ ${ }^{2}$ Université de Bordeaux, CNRS, Bordeaux Institut National Polytechnique, CBMN (UMR \\ 5248), Institut Europeen de Chimie Biologie, 2 Rue Escarpit, 33600 Pessac, France \\ ${ }^{3}$ Department Pharmazie, Ludwig-Maximilians-Universität München \\ Butenandtstraße 5 - 13 D-81377 Munich, Germany
}

* To Whom correspondence should be addressed

Jean Duhamel: jduhamel@uwaterloo.ca

Victor Maurizot: victor.maurizot@u-bordeaux.fr

Ivan Huc : ivan.huc@cup.lmu.de 


\section{ABSTRACT}

This study introduces a global fluorescence decay analysis that substantially simplifies the acquisition and analysis of time-resolved fluorescence decays acquired with a vertically polarized excitation and vertically $(\operatorname{Ivv}(t))$ and horizontally $\left(I_{\mathrm{VH}}(t)\right)$ polarized emission for time-resolved fluorescence anisotropy (TRFA) measurements. TRFA measurements were conducted whereby the $I_{\mathrm{VV}}(t)$ and $I_{\mathrm{VH}}(t)$ fluorescence decays of a series of oligoquinolines labeled at one end with an oligo(phenylene vinylene) dye (OPV-Q $\mathrm{Q}_{\mathrm{n}}$ with $\left.n=4,7,17,24,33\right)$ were acquired according to the standard protocol that is currently accepted in the scientific literature which involves toggling the emission polarizer before fitting linear combinations of the $I_{\mathrm{Vv}}(t)$ and $I_{\mathrm{VH}}(t)$ decays or acquiring the $I \mathrm{vv}(t)$ and $I \mathrm{vH}(t)$ decays with static polarizers before fitting them globally. The rotational time $(\phi)$ and initial anisotropy $\left(r_{\mathrm{o}}\right)$ retrieved from these analyses were identical within experimental error regardless of whether the decays were acquired with toggling or static polarizers and fitted according to the standard protocol or globally. These experimental results were further supported by retrieving the parameters used to generate mono-, bi-, and tri-exponential TRFAs from the global analysis of simulated $I_{\mathrm{Vv}}(t)$ and $I_{\mathrm{VH}}(t)$ fluorescence decays which were found to match perfectly the values that were inputted. Together, these experiments and simulations demonstrated that the parameters describing any type of TRFA can be extracted directly from the analysis of the $I \mathrm{Vv}(t)$ and $I_{\mathrm{VH}}(t)$ fluorescence decays acquired with a standard time-resolved fluorometer, a substantial simplification compared to the protocols currently in place to determine the TRFA. 


\section{INTRODUCTION}

Time-resolved fluorescence anisotropy (TRFA) is one of the most popular fluorescence techniques used to probe macromolecules thanks to a number of instrumental modifications implemented on a time-resolved fluorometer. ${ }^{1-3}$ Here we introduce a global fluorescence decay analysis that enables the experimentalist to perform TRFA experiments on a standard time-resolved fluorometer without the need for modifications, thus simplifying instrumentation and data acquisition protocol. TRFA is typically employed to gain detailed information at the molecular level on the internal dynamics and structure of macromolecules in solution. TRFA yields the rotational time of a macromolecule by monitoring as a function of time how a dye rigidly bonded to a macromolecule tumbles in solution. Tight binding of the dye to the macromolecule ensures that each motion of the dye is the result of a similar motion undergone by the macromolecule. In a TRFA experiment, the dye is excited with vertically polarized light and the fluorescence signal is acquired through a vertically and horizontally oriented polarizer to yield the fluorescence decays $I_{/ /}(t)$ and $I_{\perp}(t)$, respectively. In turn, the $I_{/ /}(t)$ and $I_{\perp}(t)$ fluorescence decays can be re-arranged into Equation 1 to yield the TRFA $(r(t))$.

$$
r(t)=\frac{I_{/ /}(t)-I_{\perp}(t)}{I_{/ /}(t)+2 I_{\perp}(t)}
$$

Implicit in Equation 1 is that the $I_{/ /}(t)$ and $I_{\perp}(t)$ fluorescence decays be acquired for a same number of excitation events with detection systems that would count vertically and horizontally polarized emission photons with the same efficiency. While such conditions would seem reasonable from a theoretical point of view, they are unfortunately never obeyed experimentally which has led to the implementation of a number of procedures to address these problems. First, 
the intensity of the excitation light source might fluctuate during the acquisition time of the $I_{/ /}(t)$ and $I_{\perp}(t)$ fluorescence decays. Since the expression of the function $r(t)$ in Equation 1 assumes that the $I_{/ /}(t)$ and $I_{\perp}(t)$ fluorescence decays were acquired over a same period of time with a steady excitation source, procedures were implemented to account for fluctuations in the excitation intensity. One procedure involved the design of a "T-geometry" for the time-resolved fluorometer (TRF) with two detectors facing each other and at right angle from the excitation light beam. ${ }^{4}$ In so doing, the experimental $I_{/ /}(t)$ and $I_{\perp}(t)$ fluorescence decays acquired with vertically and horizontally oriented emission polarizers, also referred to as the $I_{\mathrm{Vv}}(t)$ and $I_{\mathrm{VH}}(t)$ fluorescence decays, respectively, could be acquired simultaneously with two different detection systems which were affected in the same manner by fluctuations in the excitation source. After normalizing for the different efficiencies of the two detection systems with the $G$-factor (i.e. $I_{/ /}(t)=k \times \operatorname{Ivv}(t)$ and $\left.I_{\perp}(t)=k \times \mathrm{G} \times I_{\mathrm{VH}}(t)\right)$, the experimental intensities $I_{\mathrm{Vv}}(t)$ and $I_{\mathrm{VH}}(t)$ could be used in Equation 2 to obtain the function $r(t)$. Another procedure involved rotating the emission polarizer at short time intervals back and forth between the vertically and horizontally polarized positions to acquire the vertically polarized photons in one memory of the multichannel analyzer (MCA) and the horizontally polarized photons in another memory of the MCA in order to build the $\operatorname{Ivv}(t)$ and $I_{\mathrm{VH}}(t)$ fluorescence decays in each memory of the MCA. ${ }^{5,6}$ By selecting a time interval $(\sim 10 \mathrm{~s})$ for the rotation of the emission polarizer that would be much shorter than the time scale of the intensity fluctuations expected in the excitation source, these fluctuations could be accounted for.

$$
r(t)=\frac{I_{V V}(t)-G \times I_{V H}(t)}{I_{V V}(t)+2 G \times I_{V H}(t)}
$$


The second problem with the TRFA experimental setup is that the vertically and horizontally polarized photons do not pass through the emission monochromator with the same efficiency. ${ }^{1}$ Here again, procedures were implemented to account for this issue. One of them consisted in exciting the solution with horizontally polarized light and emission polarizers oriented vertically and horizontally to yield the $I_{\mathrm{HV}}(t)$ and $I_{\mathrm{HH}}(t)$ fluorescence decays that would both be a good representation of $I_{\perp}(t)$ differing solely from each other by the $G$-factor since $I_{\mathrm{HV}}(t)$ would equal $G \times I_{\mathrm{HH}}(t) .{ }^{6}$ Such procedures resulted in Equation 2 which has been applied for decades to represent the TRFA.

While the procedures described above are well established in the scientific literature, this report questions whether the acquisition and analysis of the $I_{\mathrm{Vv}}(t)$ and $I_{\mathrm{VH}}(t)$ fluorescence decays could be conducted in a simpler, cheaper, and more efficient manner. The procedures governing the acquisition and analysis of $\operatorname{Ivv}(t)$ and $I_{\mathrm{vH}}(t)$ fluorescence decays were implemented in the 1970's. Yet, we suspect that the main reason why these procedures were implemented in the first place was because scientists in the 1970's could not dream of the computing power available today. This report proposes a new analytical method based on the global analysis of the $I_{\mathrm{Vv}}(t)$ and $I_{\mathrm{VH}}(t)$ fluorescence decays acquired with a standard time-resolved fluorometer equipped with static polarizers. This represents a major improvement over current protocols as it simplifies substantially the study of macromolecules by TRFA by enabling such studies to be conducted with a standard time-resolved fluorometer with no add-ons besides the purchase of polarizers.

\section{EXPERIMENTAL}

Materials: The preparation and characterization of the oligoquinoline foldamers bearing an oligo(phenylene vinylene) dye have been described in details earlier. ${ }^{7-9}$ Figure 1 gives the chemical 
structure and expected conformation in solution of the OPV-Q $\mathrm{Q}_{\mathrm{n}}$ constructs. HPLC-grade chloroform from Sigma Aldrich was used in all fluorescence experiments.

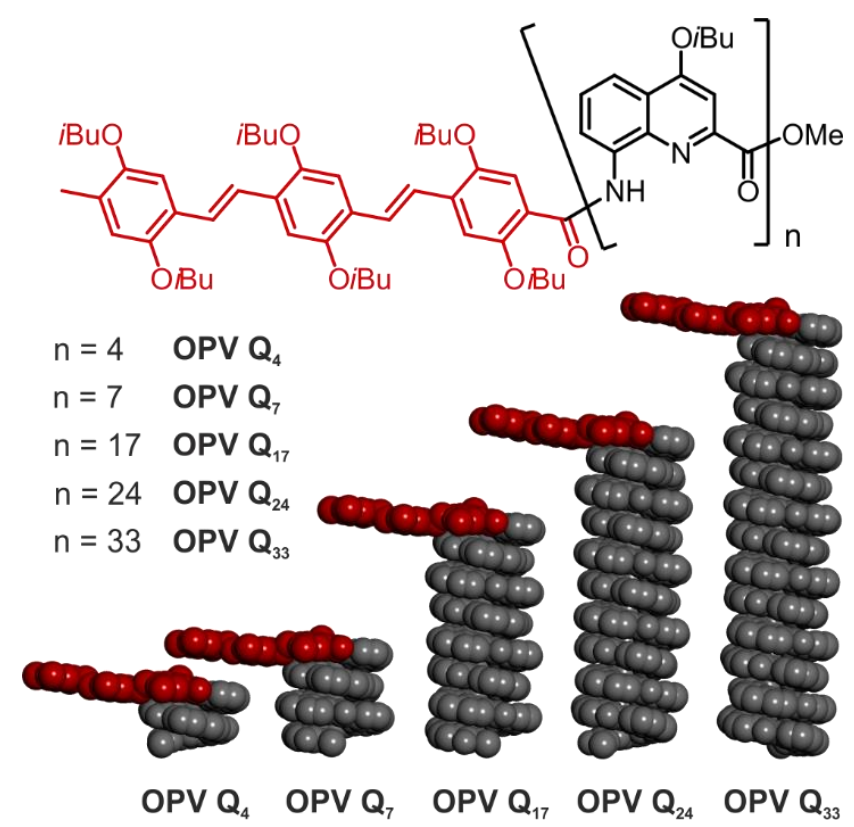

Figure 1. Chemical formula of studied oligomers and energy minimized molecular models of oligomers with the OPV unit in red to illustrate its orientation perpendicular to the helical axis. iso-Butoxy side chains and protons are omitted for clarity. ${ }^{9}$

Absorption measurements: The concentration of the OPV- $\mathrm{Q}_{\mathrm{n}}$ solutions was set to $1.4 \times 10^{-5} \mathrm{M}$ which corresponded to an absorbance of 0.09 at the excitation wavelength of $479 \mathrm{~nm}$ for the fluorescence measurements. The absorption spectra were acquired on a Cary $100 \mathrm{UV}$-vis spectrophotometer with quartz cells having a $1.0 \mathrm{~cm}$ path length.

Time-Resolved Fluorescence (TRF): All fluorescence decays were acquired in triplicate on a HORIBA DeltaFlex Fluorescence Lifetime Spectrofluorometer. Excitation was made with a delta diode laser having a maximum emission intensity at $479 \mathrm{~nm}$ and a repetition rate of $20 \mathrm{MHz}$. The 
slit width of the emission monochromator was set at $12 \mathrm{~nm}$ and the decays were obtained by monitoring the fluorescence intensity at $510 \mathrm{~nm}$. The instrument response function (IRF) was collected at $\lambda_{\mathrm{em}}=\lambda_{\mathrm{ex}}=479 \mathrm{~nm}$ using an aluminium prism to reflect the excitation beam to the detector. The natural lifetime $\left(\tau_{\mathrm{o}}\right)$ of the OPV dye was determined by fitting the fluorescence decays $(\operatorname{IVM}(t))$ of the OPV-Q and the emission polarizer set at the magic angle $\left(54.7^{\circ}\right)$ with a single exponential yielding a $\tau_{\mathrm{o}}$ value of $1.61 \pm 0.05 \mathrm{~ns}$. The TRFA experiments were conducted in three different manners. The first procedure applied the standard protocol and used the programs provided with the HORIBA fluorometer. The OPV-Q $\mathrm{Q}_{\mathrm{n}}$ solutions were excited with vertically polarized photons and the emission polarizer was toggled back and forth between the vertical and horizontal position to yield the $I_{\mathrm{Vv}}(t)$ and $I_{\mathrm{VH}}(t)$ fluorescence decays. The acquisition was conducted until the $I_{\mathrm{Vv}}(t)$ decays had 10,000 more counts at their maximum than the $I_{\mathrm{VH}}(t)$ decays thus ensuring that the $I_{\mathrm{VH}}(t)$ decays had more than 10,000 counts at their maximum. The same experiments were repeated with a horizontally polarized excitation to yield the $I_{\mathrm{HV}}(t)$ and $I_{\mathrm{HH}}(t)$ decays which were employed to determine the $G$-factor. The $I \mathrm{Vv}(t)$ and $I_{\mathrm{VH}}(t)$ decays were combined with the $G$-factor to calculate first, the sum $I_{\mathrm{S}}(t)=I_{\mathrm{Vv}}(t)+2 G \times I_{\mathrm{VH}}(t)$, i.e. the denominator of Equation 2, and second, the difference $I_{\mathrm{D}}(t)=I_{\mathrm{Vv}}(t)-\mathrm{G} \times I_{\mathrm{VH}}(t)$, i.e. the numerator of Equation 2. $I_{\mathrm{S}}(t)$ was fitted to a sum of exponentials to characterize the natural fluorescence decay of the OPV without polarization effects. $I_{\mathrm{D}}(t)$ was compared to the convolution product of the IRF and the product $I_{\mathrm{S}}(t) \times r(t)$ where the parameters describing $I_{\mathrm{S}}(t)$ were fixed in the analysis and $r(t)$ was given by Equation 3 to determine the rotational time $(\phi)$ and initial anisotropy $\left(r_{\mathrm{o}}\right)$ of the OPV-Q $\mathrm{Q}_{\mathrm{n}}$ sample. ${ }^{10}$ This procedure reflected standard protocols that are accepted in the scientific literature for the determination of the TRFA as this procedure requires the prior determination of the $G$-factor. ${ }^{1-6}$ 


$$
r(t)=r_{o} \times \exp (-t / \phi)
$$

The second procedure fitted the $I_{\mathrm{vv}}(t)$ and $I_{\mathrm{VH}}(t)$ fluorescence decays acquired with toggling polarizers globally according to Equations 4 and 5, respectively, using the program aniso01c that optimizes the $G$-factor. ${ }^{9}$ The third procedure consisted in acquiring the $I \mathrm{vv}(t)$ and $I_{\mathrm{VH}}(t)$ fluorescence decays with a vertically polarized excitation and a static emission polarizer set first in the vertical position and then in the horizontal position until both decays reached 10,000 counts at their maximum. The $I_{\mathrm{Vv}}(t)$ and $I_{\mathrm{VH}}(t)$ fluorescence decays were then fitted globally with the program aniso01c. The parameters were optimized according to the Marquardt-Levenberg algorithm. ${ }^{11}$

$$
\begin{gathered}
I_{V V}(t)=\frac{I_{o}}{3} e^{-t / \tau_{o}} \times(1+2 r(t)) \\
I_{V H}(t)=\frac{I_{o}}{3 G} e^{-t / \tau_{o}} \times(1-r(t))
\end{gathered}
$$

Simulations: To assess the generality of the analysis that optimizes the $G$-factor, $I_{\mathrm{vv}}(t)$ and $I_{\mathrm{VH}}(t)$ fluorescence decays were simulated by convoluting an IRF with Equations 4 and 5, respectively, and adding Poisson noise to the simulated decays. Mono-, bi-, and tri-exponential $r(t)$ functions shown in Equations 3 and S3 and S4 in Supporting Information (SI) were employed to represent the TRFA for OPV-labeled foldamers ${ }^{9}$ and DNA duplexes with intercalated ethidium bromide that would form an angle of 90 or $75^{\circ}$ with respect to the main helix axis, ${ }^{12}$ respectively. For each macromolecular construct, $20 I_{\mathrm{Vv}}(t)$ and $20 I_{\mathrm{VH}}(t)$ fluorescence decays were simulated with 
different patterns of Poisson noise and 5 different $G$-factor values in order to assess the effect of the value of the $G$-factor on the parameters retrieved from the analysis. A detailed description of the decay simulations is provided in SI.

\section{RESULTS AND DISCUSSION}

The procedures described in the Introduction were implemented to normalize the fluorescence intensities $I_{\mathrm{Vv}}(t)$ and $I_{\mathrm{VH}}(t)$ with respect to each other so that Equation 2 would become equivalent to Equation 1. Yet Equations 1 and 2 were implemented originally to bring under a single equation (i.e. Equation 2) the information about $r(t)$ that was contained in the $I_{\mathrm{Vv}}(t)$ and $I_{\mathrm{VH}}(t)$ fluorescence decays as illustrated in Equations 4 and 5. Equations 4 and 5 make abundantly clear that the $G$ factor is only a scaling factor which could be easily optimized.

The normalization of two fluorescence decays describing a same photophysical process is far from being a novel concept. The global analysis of fluorescence decays has been quietly applied for the past 15 years by this laboratory to study first, pyrene excimer formation in pyrene-labeled macromolecules ${ }^{13,14}$ and second, the radiationless transfer of energy from an excited pyrene end group to the core of dendronized porphyrins. ${ }^{15,16}$ These studies were enabled by a normalization process that was inherent to the global analysis of the fluorescence decays of the pyrene monomer and excimer on the one hand or of the pyrene donor and the porphyrin acceptor on the other hand. The mathematical procedure consisting in normalizing two fluorescence decays describing a same photophysical phenomenon is now applied to the $I_{\mathrm{Vv}}(t)$ and $I_{\mathrm{VH}}(t)$ fluorescence decays.

To this end, the $I_{\mathrm{Vv}}(t)$ and $I_{\mathrm{VH}}(t)$ fluorescence decays were acquired first, with toggling polarizers until the $I_{\mathrm{vv}}(t)$ decay maximum had 10,000 more counts than the $I_{\mathrm{VH}}(t)$ decay maximum and second, with static polarizers until both the $I_{\mathrm{Vv}}(t)$ and $I_{\mathrm{VH}}(t)$ decay maxima reached 10,000 
counts, for a series of OPV-Q $\mathrm{Q}_{\mathrm{n}}$ constructs with $n=4,7,17,24$, and 33 . The synthesis and characterization of the OPV-Q foldamers have already been described in earlier publications. ${ }^{7-9}$ In particular, the $I_{\mathrm{Vv}}(t)$ and $I_{\mathrm{VH}}(t)$ fluorescence decays of the OPV-Q foldamers acquired previously with static polarizers were found to be well-fitted according to Equations 4 and 5 by assuming a monoexponential TRFA as shown in Equation 3 where $r_{\mathrm{o}}$ and $\phi$ are the initial anisotropy and the rotational time, respectively. ${ }^{9}$ More information about the acquisition of the fluorescence decays and their fits, which were excellent, according to the different protocols can be found in SI.

Figure 2 shows the parameters $\phi, r_{\mathrm{o}}$, and $G$ obtained by fitting the $I_{\mathrm{Vv}}(t)$ and $I_{\mathrm{VH}}(t)$ fluorescence decays that were first, acquired with toggling polarizers and analyzed with the standard protocol that calculates the $G$-factor, second, acquired with the toggling polarizers and globally analyzed with optimization of the $G$-factor, and third, acquired with static polarizers and globally analyzed with optimization of the $G$-factor. The parameters $\phi$ and $r_{\mathrm{o}}$ shown in Figure $2 \mathrm{~A}$ and $\mathrm{B}$, respectively, were identical within error bars regardless of the procedure applied. The rotational time $\phi$ increased linearly with increasing number of units, passing through a non-zero intercept corresponding to the rotational time of the OPV moiety $(\phi=0.27 \pm 0.02 \mathrm{~ns}) .{ }^{9}$ This behavior was taken as an indication that any addition of a quinoline unit increased the hydrodynamic volume of the OPV-Q $\mathrm{Q}_{\mathrm{n}}$ constructs by a set increment as would be expected from rigid helical objects in solution. The initial anisotropy, $r_{\mathrm{o}}$, decreased slightly from 0.40 , corresponding to the $r_{\mathrm{O}}$ value of OPV, to 0.33 for OPV-Q ${ }_{33}$ indicating some wobbling of the OPV moiety with respect to the helical foldamer. These results were in perfect agreement with those presented in an earlier publication. ${ }^{9}$ Within experimental error, the $G$-factor of $0.88 \pm 0.04$ obtained experimentally through the analysis of the $I_{\mathrm{HV}}(t)$ and $I_{\mathrm{HH}}(t)$ fluorescence decays and used to fit the 
$I_{\mathrm{Vv}}(t)$ and $I_{\mathrm{VH}}(t)$ fluorescence decays acquired with the toggling polarizers according to the standard protocol was identical to the G-factor of $0.92 \pm 0.05$ optimized with our analysis program aniso01c as shown in Figure 2C. Since the $I_{\mathrm{VH}}(t)$ fluorescence decays acquired with static or toggling polarizers did not have the same number of counts at their decay maxima, global analysis of the $I_{\mathrm{Vv}}(t)$ and $I_{\mathrm{VH}}(t)$ fluorescence decays with both 10,000 counts at the decay maximum and acquired with static polarizers yielded a different G-factor whose value of $0.42 \pm 0.01$ remained the same for all OPV-Q $\mathrm{Q}_{\mathrm{n}}$ samples.

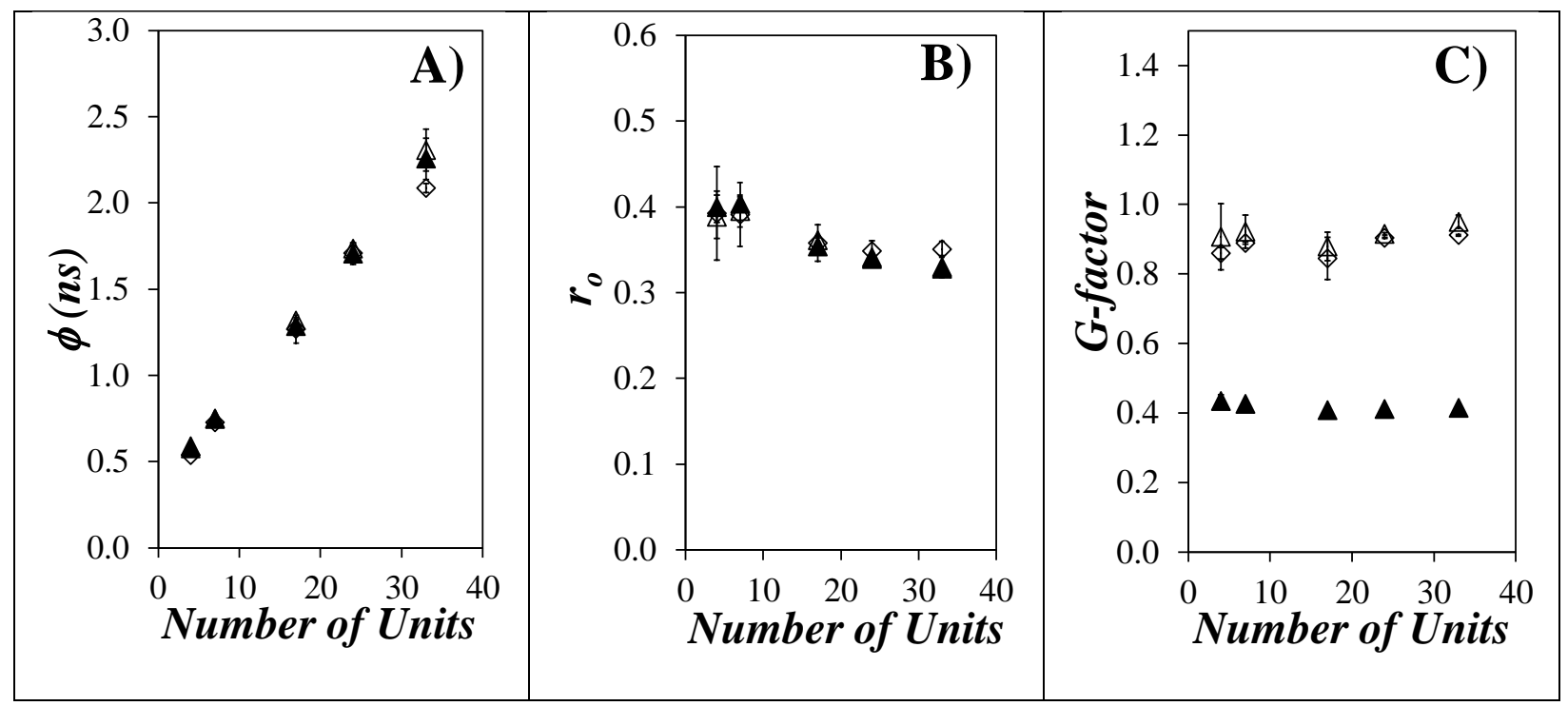

Figure 2. Plots of A) rotational times ( $\phi$ ), B) initial anisotropy $\left(r_{\mathrm{o}}\right)$, and C) G-factor as a function of the number of quinoline units. The $I_{\mathrm{Vv}}(t)$ and $I_{\mathrm{VH}}(t)$ decays were acquired $(\mathbf{\Delta})$ up to 10,000 counts for both decays with static polarizers and $(\boldsymbol{\Delta}, \boldsymbol{\nabla})$ with a 10,000 counts difference between the $I_{\mathrm{Vv}}(t)$ and $I_{\mathrm{VH}}(t)$ decays with toggling polarizers. The $I_{\mathrm{Vv}}(t)$ and $I_{\mathrm{VH}}(t)$ decays acquired with ( A) static and $(\boldsymbol{\Delta})$ toggling polarizers were fitted with our fluorescence decay analysis program and the $I_{\mathrm{Vv}}(t)$ and $I_{\mathrm{VH}}(t)$ decays acquired with $(\diamond)$ toggling polarizers were fitted according to the HORIBA protocol. 
To further strengthen the conclusion that global analysis of $I_{\mathrm{Vv}}(t)$ and $I_{\mathrm{VH}}(t)$ fluorescence decays with optimization of the $G$-factor yielded parameters that reflected the geometric features of a symmetric top macromolecular object (STMO) that might have one (for a sphere, see Equation 3) or two or three (for a STMO, see Equation S1 in SI) ${ }^{12}$ rotational times, a series of $20 I_{\mathrm{Vv}}(t)$ and $20 I_{\mathrm{VH}}(t)$ fluorescence decays were simulated with 20 different Poisson noise patterns for mono(Equation 3), bi- (Equation S3), and tri- (Equation S4) exponential TRFAs. The decays simulated with Equation 3 using the parameters obtained experimentally for OPV-Q ${ }_{n}$ foldamers are discussed first.

Assuming a constant $r_{\mathrm{o}}$ value of 0.4 and that $\phi$ increased linearly with the number of quinoline units $(N U)$ according to Equation $\mathrm{S} 2$, the $I_{\mathrm{vv}}(t)$ and $I_{\mathrm{vH}}(t)$ fluorescence decays were simulated for hypothetical OPV-Q $\mathrm{Q}_{\mathrm{n}}$ constructs and different $G$-factors before being fitted globally by optimizing the $G$-factor. The retrieved parameters $\left(\phi, r_{\mathrm{o}}\right.$, and $\left.G\right)$ were plotted in Figure 3A and $\mathrm{B}$ and were in excellent agreement with the inputted values. The calculated $\phi$ and $r_{\mathrm{o}}$ values in Figure 3A were averaged over all the $G$-factors for which simulated decays were prepared for a given $N U$, thus representing 100 pairs of simulated $I_{\mathrm{Vv}}(t)$ and $I_{\mathrm{VH}}(t)$ fluorescence decays for each data point. The calculated values in Figure 3B were averaged over the 20 pairs of $I_{\mathrm{Vv}}(t)$ and $I_{\mathrm{VH}}(t)$ fluorescence decays simulated for a given $G$-factor. The trends shown in Figure 3 clearly illustrated that the parameters $r_{\mathrm{o}}$ and $\phi$ retrieved from a global analysis of simulated $I_{\mathrm{Vv}}(t)$ and $I_{\mathrm{VH}}(t)$ fluorescence decays that optimized the $G$-factor were identical within minuscule error bars to the inputted values regardless of the $G$-factor used in the decay simulation. Excellent agreement was also obtained between the inputted and retrieved $G$-factors in Figure 3B. 


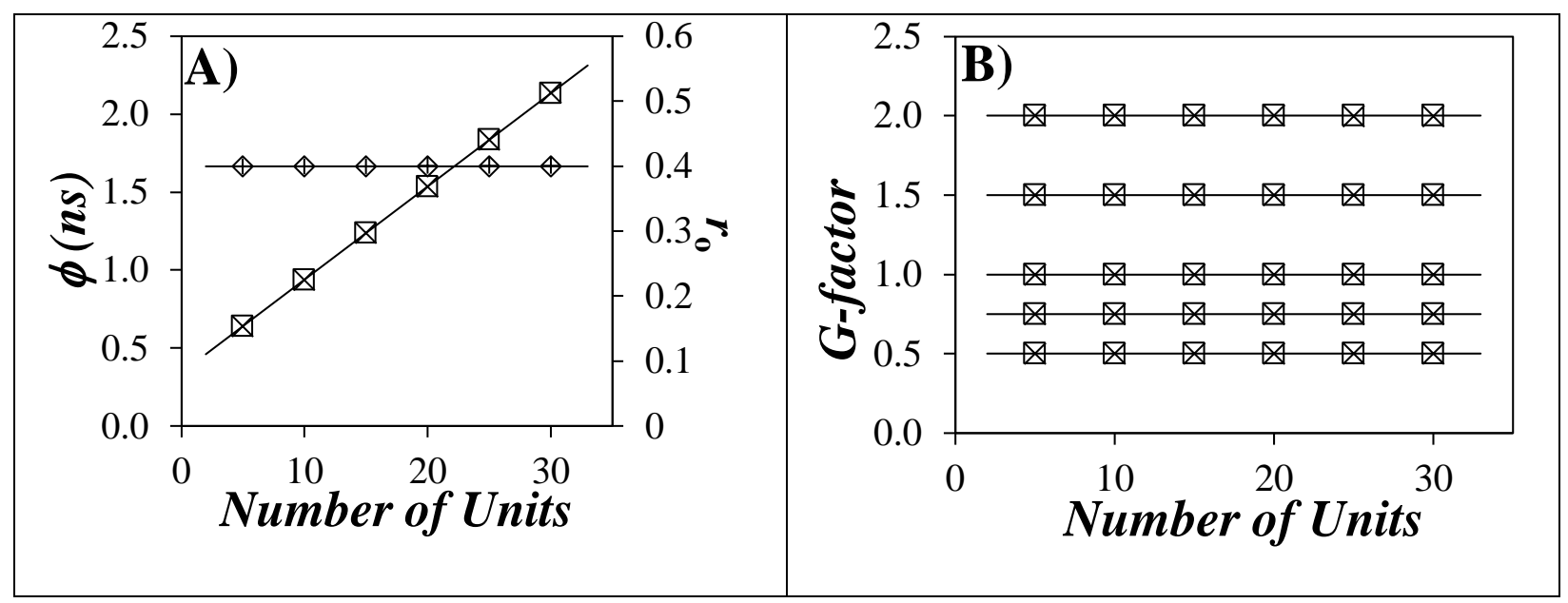

Figure 3. Plots of the $(\boldsymbol{\square}, \boldsymbol{\nabla})$ inputted and calculated $(\times,+)$ values of $A)(\boldsymbol{\square}, \times) \phi$ and $(\boldsymbol{\nabla},+) r_{0}$, and C) $G$-factor plotted as a function of the number of quinoline units.

Since the TRFA of the OPV-Q foldamers with $n$ between 4 and 33 (see Figure 1) were well-represented by a single rotational time, these TRFAs could not be used to generate bi- and tri-exponential TRFAs that required two and three distinct rotational times. To obtain bi- and triexponential TRFAs, the parameters obtained from an earlier study for the anisotropy of ethidium bromide intercalated between base pairs of DNA duplexes were used as described in SI. ${ }^{\text {Erreur }}$ ! Signet non défini. The $I_{\mathrm{Vv}}(t)$ and $I_{\mathrm{VH}}(t)$ decays were simulated using first the biexponential TRFA given in Equation S3. The resulting 700 decays were fitted with aniso02n-4 and the wobbling angle $l$ and diffusion coefficients $D_{/ /}$and $D_{\perp}$ retrieved from the analysis were plotted in Figure 4 A. The agreement between inputted and retrieved $D_{/ /}, D_{\perp}$, and $l$ values was excellent. The corresponding $G$-factors are presented in Figure 4B. As for the $D_{/ /}, D_{\perp}$, and $l$ parameters, the values retrieved for the $G$-factors from the analysis agreed very well with the inputted values. The $I_{\mathrm{Vv}}(t)$ and $I_{\mathrm{VH}}(t)$ decays simulated by assuming a tri-exponential TRFA as described in Equation S4 were fitted and the $D_{/ /}, D_{\perp}$, and $l$ parameters and $G$-factors retrieved from this analysis resulted in plots shown in Figure S4 in SI that were identical to the plots in Figure 4. This outcome was expected since the 
fits of the $I_{\mathrm{vv}}(t)$ and $I_{\mathrm{VH}}(t)$ decays simulated with a bi- and tri-exponential TRFA used a same number of floating parameters in the optimization routine, and thus retrieved these same parameters with a similar accuracy.

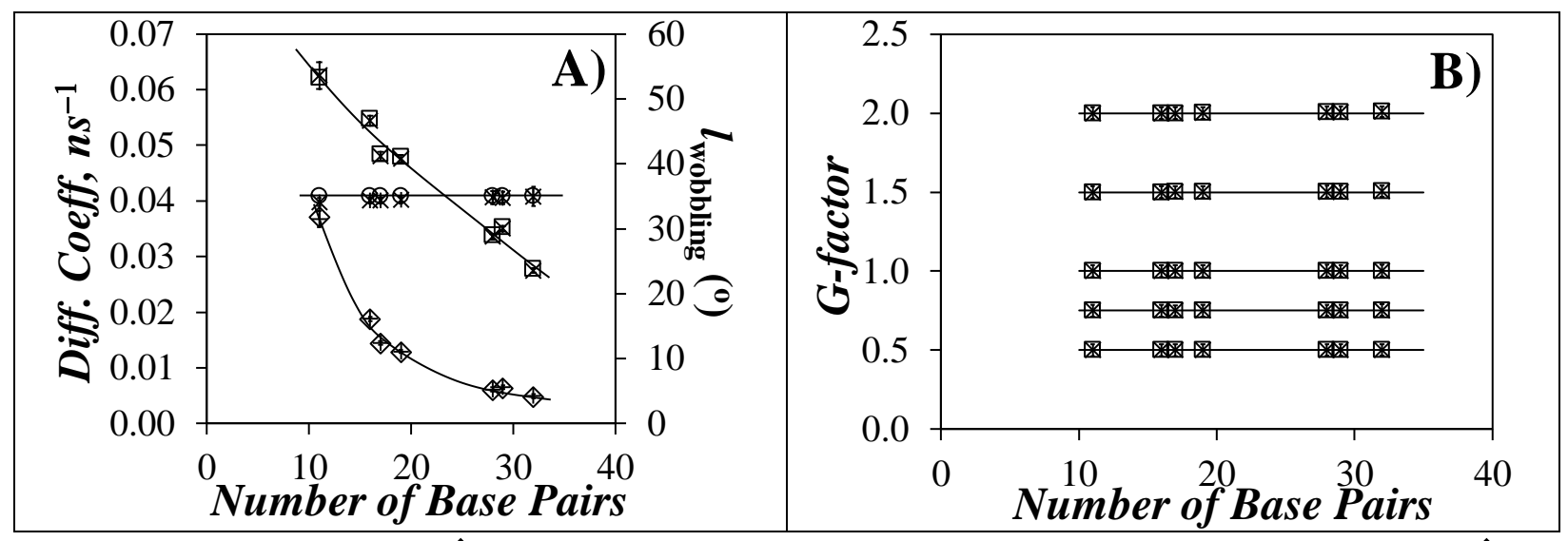

Figure 4. Plots of the $(\boldsymbol{\square}, \diamond, \mathbf{0})$ inputted and $(\times,+, *)$ calculated values of $A)\left(\boldsymbol{\square}_{, \times}\right) D_{/ /},\left(\diamond_{,+}\right)$ $D_{\perp}$, and $(\mathbf{O}, *) r_{\mathrm{o}}$, and C) $G$-factor plotted as a function of the number of DNA base pairs. Biexponential TRFA obtained as described in the Experimental section.

Allowing all $\left(r_{\mathrm{i}}\right)$ pre-exponential factors and $\left(D_{/ /}\right.$and $\left.D_{\perp}\right)$ diffusion coefficients in the TRFA to float in the analysis resulted in poorer accuracy for the individual parameters, but the initial anisotropy $\left(r_{0}=\Sigma r_{\mathrm{i}}\right)$, average rotational time $\langle\phi\rangle$, and $G$-factor were retrieved with excellent accuracy. These additional studies are discussed in details in SI. All together, these studies demonstrate that the $G$-factor can always be optimized in the analysis of $I_{\mathrm{Vv}}(t)$ and $I_{\mathrm{VH}}(t)$ fluorescence decays regardless of whether the TRFA is a mono-, bi-, or tri-exponential function. 


\section{CONCLUSIONS}

The experimental and simulated data provided in this report suggest that the $I_{\mathrm{Vv}}(t)$ and $I_{\mathrm{VH}}(t)$ fluorescence decays acquired with static polarizers and fitted globally with a program that optimizes the $G$-factor as one of the floating parameters yield the same values for the parameters used to describe the TRFA that would be obtained by a much more complex procedure..$^{4-6,10,12}$ This latter procedure corresponds to currently accepted protocols that are applied to obtain the parameters describing the TRFA and it requires a specialized time-resolved fluorometer. In contrast, the proposed procedure only requires a standard time-resolved fluorometer, an excitation and emission polarizer, and a global analysis program. This procedure will enable scientists, who do not have access to a specialized time-resolved fluorometer, to conduct TRFA experiments with their standard instrument, a notable improvement. Possibly even more important, this study further supports the claim that global analysis of two fluorescence decays probing two different features of a same photophysical process, be it the pyrene monomer and excimer decays for excimer formation, the energy donor and acceptor decays for FRET, or the $I_{\mathrm{Vv}}(t)$ and $I_{\mathrm{VH}}(t)$ decays for TRFA measurements, requires an inherent mathematical normalization of the fluorescence decays which enables the study of these complex photophysical processes with a standard time-resolved fluorometer.

\section{ACKNOWLEDGMENTS}

HL, JW, and JD thank a collaborative funding opportunity from the University of Waterloo and NSERC.

\section{SUPPORTING INFORMATION}


Detailed description of the simulations and the fits of the experimental and simulated $I_{\mathrm{Vv}}(t)$ and $I_{\mathrm{VH}}(t)$ decays, parameters retrieved from the analyses of the experimental fluorescence decays, listings of analysis programs written in Fortran.

\section{REFERENCES}

1. Lakowicz, J. R. Principles of Fluorescence Spectroscopy, 3rd Ed. Springer, 2006, pp 383-441.

2. Valeur, B.; Nuno Berberian Santos, M. Molecular Fluorescence : Principles and Applications, 2nd Ed. Wiley, 2012, pp 181-212.

3. Jameson, D. M. Introduction to Fluorescence. CRC Press, 2014, pp 75-99.

4. Schuyler, R.; Isenberg, I. A Monophoton Fluorometer with Energy Discrimination. Rev. Sci. Inst. 1971, 42, 813-817.

5. Dale, R. E.; Chen. L. A.; Brand, L. Rotational Relaxation of the "Microviscosity" Probe Diphenylhexatriene in Paraffin Oil and Egg Lecithin Vesicles. J. Biol. Chem.1977, 252, 75007510.

6. Wahl, Ph. Analysis of Fluorescence Anisotropy Decays by a Least Square Method. Biophys. Chem. 1979, 10, 91-104.

7. Li, X.; Markandeya, N.; Jonusauskas, G.; McClenaghan, N. D.; Maurizot, V.; Denisov, S. A.; Huc, I. Photoinduced electron transfer and hole migration in nanosized helical aromatic oligoamide foldamers J. Am. Chem. Soc. 2016, 138, 13568-13578.

8. Wang, X.; Wicher, B.; Ferrand, Y.; Huc, I. Orchestrating Directional Molecular Motions: Kinetically Controlled Supramolecular Pathways of a Helical Host on Rodlike Guests. J. Am. Chem. Soc. 2017, 139, 9350-9358. 
9. Wang, J.; Little, H.; Duhamel, J.; Li, X.; Nagula, M.; Maurizot, V.; Huc, I. Application of Time-Resolved Fluorescence Anisotropy to Probe Quinoline-Based Foldamers Labeled with Oligo(phenylene-vinylene). Macromolecules 2019, 52, 5829-5837.

10. Barkley, M. D.; Kowalczyk, A. A.; Brand, L. Fluorescence Decay Studies of Anisotropic Rotations of Small Molecules. J. Chem. Phys. 1981, 75, 3581-3593.

11. Press, W. H.; Flanery, B. P.; Tenkolsky, S. A.; Vetterling, W. T. Numerical Receipes in Fortran: The Art of Scientific Computing, Cambridge University Press, Cambridge, Endgland and New York, 1992, pp 523-528.

12. Duhamel, J.; Kanyo, J.; Dinter-Gottlieb, G.; Lu, P. Fluorescence Emission of EB intercalated in Defined DNA Duplexes: Hydrodynamics and Base Pair Twisting Components. Biochemistry 1996, 35, 16687-16697.

13. Siu, H.; Duhamel, J. Global Analysis of the Fluorescence Decays of a Pyrene-Labelled Polymer Using a Blob Model. Macromolecules 2004, 37, 9287-9289.

14. Duhamel, J. Global Analysis of Fluorescence Decays to Probe the Internal Dynamics of Fluorescently Labeled Macromolecules. Langmuir 2014, 30, 2307-2324.

15 . Zaragoza-Galán, G.; Fowler, M.; Duhamel, J.; Rein, R.; Solladié, N.; Rivera, E. Synthesis and Characterization of Novel Pyrene-Dendronized Porphyrins Exhibiting Efficient Fluorescence Resonance Energy Transfer (FRET): Optical and Photophysical Properties. Langmuir 2012, $28,11195-11205$.

16. Zaragoza-Galán, G.; Fowler, M.; Rein, R.; Solladié, N.; Duhamel, J.; Rivera, E. Fluorescence Resonance Energy Transfer in Partially and Fully Labeled Pyrene Dendronized Prophyrins Studied with Model Free Analysis. J. Phys. Chem. C 2014, 118, 8280-8294. 


\section{Table of Contents}

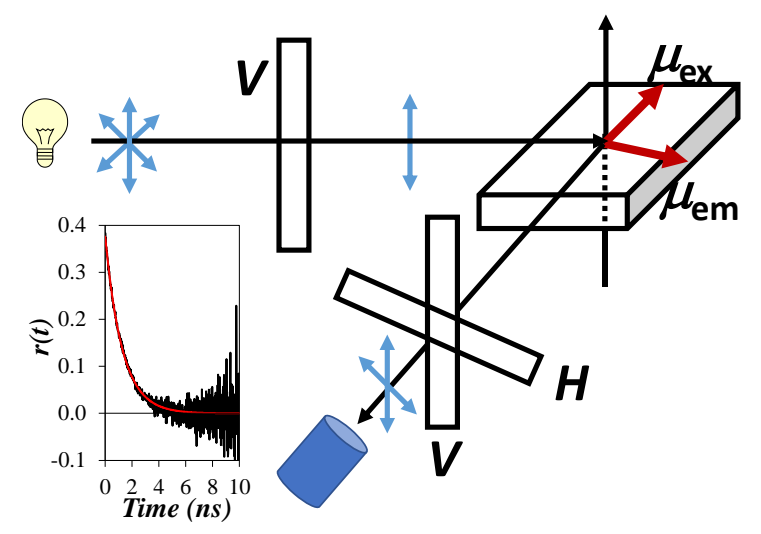

